\title{
Efficiency in Carrying Cargo to Earth Orbits: Spaceports Repositioning
}

\section{Jakub Hospodka ${ }^{1 *}$, Zdeněk Houfek ${ }^{1}$}

${ }^{1}$ Department of Air Transport, Faculty of Transportation Sciences, Czech Technical University in Prague, Prague,

Czech Republic

*Corresponding author: Czech Technical University in Prague, Faculty of Transportation Sciences, Department of Air

Transport, Horská 3, 12803 Prague, Czech Republic, Email: xhospodka@fd.cvut.cz

\begin{abstract}
Space flights are in these days not any more question of technology, but more question of costs. One way how to decrease cost of launch is change of home spaceport. Change of home spaceport for different rockets is a way to achieve more efficient launches to space. The reason is different acceleration achieved from Earth rotation. We added several mathematical calculations of missions to Low Earth Orbit and Geostationary Earth Orbit to show bonuses from Earth rotation and effect of atmospheric drag on specific rockets used these days. We discussed only already used space vessels. Namely Arianne 5, Delta 4 heavy, Proton-M, Zenit and Falcon9. For reaching GEO we discuss possibility of using Hohmman transfer, because none of aforementioned vessels is available for direct GEO entry. As possible place for launch we discussed spaceports Baikonur, Kennedy Space center, Guyana Space center and Sea Launch platform. We present results in form of additional acceleration for each spaceport, and we also project this additional acceleration in means payload increase. In conclusion we find important differences between vessel effectivity based on spaceport used for launch. Change of launch location may bring significant cost decrease for operators.
\end{abstract}

\section{Keywords}

Atmosferic drag - Earth rotation - Hohmann transfer - Rockets - Spaceports - Tsiolkovski equation

\section{Introduction}

Currently, missions to Earth orbits and especially to GEO are made from few locations on Earth. These spaceports are normally bound to the national programs or agencies and only part of its launches are made for commercial purposes. In these days there are some spaceports designed purely for commercial purposes, but due to the cost of these spaceports they are very few in numbers.

Economic efficiency in using rockets from spaceports owned by their national agency is the main task of this article.
Space missions to Low Earth Orbit (LEO) and Geostationary Earth orbit (GEO) from four different spaceports placed on different latitudes are representing all possible economic outputs. Because of increased use of orbits and deep space the economic point of view due to huge cost of rocket launch is coming more to the light of the day. Exploration of our solar system or for example mining resources on asteroids will be possible only if we manage to decrease the cost of space flights. Efficiency in using spaceports on our planet is one of the first steps we need to take to conquer our so- 
lar system. Better spaceport efficiency is based additional speed added from rotation of Earth. This additional velocity raises from poles to equator. Obviously this effect only occurs for launches for GEO of LEO without inclination, and only launches made eastbound.

\section{Current spaceports}

To show difference in spaceports we use these days four representatives were chosen:

1. Baikonur - Russian main spaceport located in Kazakhstan representing the northernmost spaceport used for high orbits and deep space missions.

2. Kennedy Space Center - NASA main spaceport located in Cape Canaveral, FL and used for all heavy and manned launches to space (KSC).

3. Guyana Space Center - ESA main spaceport located in French Guyana, currently the biggest Earth placed spaceport nearest to Equator (GSC).

4. Sea Launch Platform - Commercial spaceport located on Equator in Pacific Ocean on an old oil mining platform reconstructed to launch rockets.

Due to rotation of Earth every missile launch in eastward direction gets bonus in speed depending on the latitude of the spaceport. Because the initial bonus on Equator is $463 \mathrm{~ms}^{-1}$ and is decreasing to the Poles we need to compensate for spaceports mentioned above with following equation:

$$
v=v_{0} \times \cos \phi
$$

Table 1. Spaceport rotation bonuses.

\begin{tabular}{lc} 
Spaceports & Rotation bonus $\left(\mathbf{m s}^{-1}\right)$ \\
\hline Baikonur & 322 \\
\hline GSC & 461 \\
\hline KSC & 407 \\
\hline Sea launch & 463 \\
\hline
\end{tabular}

\section{Rocket performance}

It is obvious that not only spaceports are responsible for economy of the space missions. It is also necessary to include rocket performance, atmospheric drag and other factors which are affecting rocket launches from Earth [1].

Five types of rockets are used in this paper to show different performance from home spaceports. These rockets are:

- Ariane 5 [2] - ESA rocket, Guyana Space Center is home spaceport

- Delta IV Heavy [3] - NASA rocket, Kennedy Space Center is home spaceport
- Falcon v1.1 [4] - SpaceX rocket, first private company rocket used to supply International Space Station (ISS), Kennedy Space Center is home spaceport

- Proton - M [5] - Russian heavy rocket, Baikonur is home spaceport

- Zenit [6] - Ukrainian rocket used by Sea Launch consorcium, Sea Launch platform is used as home spaceport

\subsection{Atmospheric drag}

Calculation of atmospheric drag depends can be made by following equation:

$$
F_{D}=\frac{1}{2} C \rho A v^{2}
$$

where $F_{D}$ is drag force, $C$ is drag coeficient, $\rho$ is the atmospheric density and $v$ is the flow velocity relative to the object.

For this equation we will use 0.5 as a drag coefficient for cone, atmospheric density in $5.5 \mathrm{~km}$ above ground and rocket speed at point of maximal dynamic pressure. A is representing referential area of the rockets which is defined by the construction. After we manage to calculate drag force from Eq. (2) it is possible to use this force with time to reach the point of maximal dynamic pressure in Eq. (3).

$$
\Delta v=\int_{0}^{t} \frac{F_{D}}{t} d t
$$

The results from integration using the launching mass of the rockets are presented in Tab. 2.

Table 2. Rockets characteristic velocities.

\begin{tabular}{lr}
\multicolumn{1}{c}{ Rocket } & $\Delta v\left(\mathbf{m s}^{-1}\right)$ \\
\hline Ariane 5 & 38,557 \\
\hline Delta IV Heavy & 106,652 \\
\hline Falcon 9 v1.1 & 24,818 \\
\hline Proton-M & 151,917 \\
\hline Zenit & 26,583 \\
\hline
\end{tabular}

\subsection{Rocket equations}

It is also necessary to get some reference numbers of speed, which is needed to get to orbits. In this case Tsiolkovski equations (see, Eq. (4)) calculating maximum possible speed for multistage rockets is good to show the difference in shifting rockets to different spaceports.

$$
\Delta v=v_{e} \ln \frac{M_{0}}{M_{1}}
$$

This equation using natural logarithm with mass ratio of start $\left(M_{0}\right)$ and final mass $\left(M_{1}\right)$ needs to be adjusted for every stage of the rocket. Exhaust velocity for each stage of rocket depends on fuel type used in the rocket. This velocity can be 
calculated from Specific impulse $I_{S P}$ with Eq. (5) using gravitational constant, which can be found in technical manuals of each rocket.

$$
v_{e}=I_{S P} \times g_{0}
$$

Also payload must be included in the mass ratio to get correct results so calculations are made with 10 tones for LEO and 4.85 tones to GEO.

In tables 3 and 4 are shown results of Tsiolkovski equations for LEO and GEO. Because each rocket has different number of stages and some have even rocket boosters on sides which needs to have another equation in following tables are shown only final results.

Table 3. Tsiolkovsky equations for LEO.

\begin{tabular}{lr}
\multicolumn{1}{c}{ Rocket } & $\Delta v\left(\mathbf{k m ~ s}^{-1}\right)$ \\
\hline Ariane 5 & 9.661 \\
\hline Delta IV Heavy & 12.013 \\
\hline Falcon 9 v1.1 & 9.705 \\
\hline Proton-M & 11.456 \\
\hline Zenit & 10.131 \\
\hline
\end{tabular}

Table 4. Tsiolkovsky equations for GEO.

\begin{tabular}{lr}
\multicolumn{1}{c}{ Rocket } & $\Delta v\left(\mathbf{k m ~ s}^{-1}\right)$ \\
\hline Ariane 5 & 10.635 \\
\hline Delta IV Heavy & 13.777 \\
\hline Falcon 9 v1.1 & 10.845 \\
\hline Proton-M & 13.041 \\
\hline Zenit & 11.515 \\
\hline
\end{tabular}

\subsection{Hohmann transfer}

Reaching GEO is possible with two options, first going directly and using more fuel in this process, and second follow a maneuver called Hohmann transfer, see Fig. 1.

For spaceports used in this paper non-coplanar Hohmann transfer (see [7]) must be used except for Sea Launch platform. Equations for this version of Hohmann transfer are:

$$
\begin{gathered}
\Delta v_{1}=\sqrt{v_{1}+v_{C S_{1}}-2 \times v_{1} \times v_{C S_{1}} \times \cos \Phi_{1}} \\
\Delta v_{2}=\sqrt{v_{2}+v_{C S_{2}}-2 \times v_{2} \times v_{C S_{2}} \times \cos \Phi_{2}} \\
\Delta v=\Delta v_{1}+\Delta v_{2}
\end{gathered}
$$

Circular speed of initial and final orbit is needed in Eq. (6) and Eq. (7) as well as apogee and perigee speed of the transfer orbit to determine exact velocity change. In technical manuals of each rocket is also specified the inclination change in first burst and in the second one to achieve the most efficient way to reach GEO.

Results from calculation are shown in Tab. 5. Small difference is noticeable between Falcon 9 and Delta IV Heavy

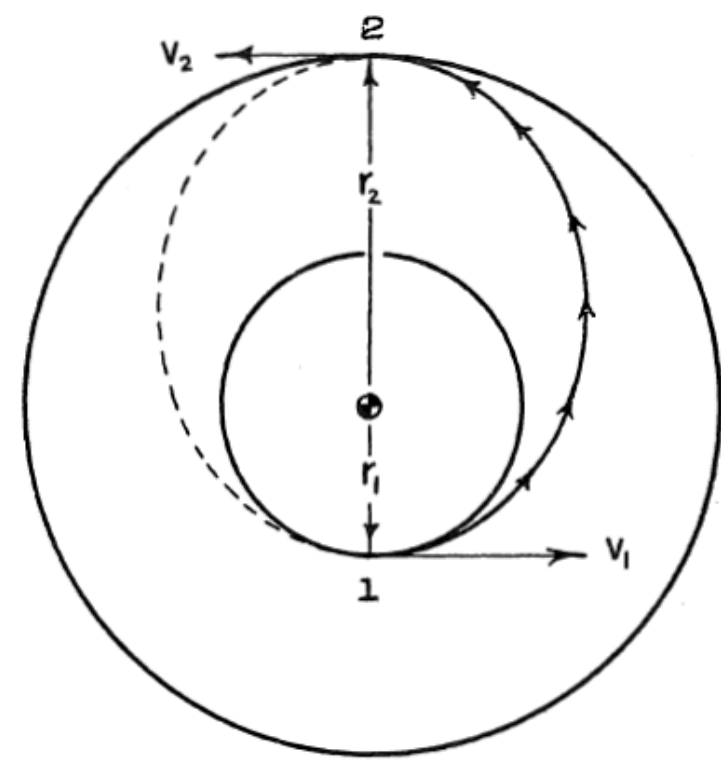

Figure 1. The model of basic objective classes.

Table 5. Results for spaceport and rockets.

\begin{tabular}{lrr} 
Spaceport/Rockets & $\begin{array}{c}\Delta \mathbf{v} \text { difference } \\
\left(\mathbf{m s}^{-1}\right)\end{array}$ & $\begin{array}{c}\text { Payload } \\
\text { increase (kg) }\end{array}$ \\
\hline GSC/Ariane 5 & 2 & 12 \\
\hline KSC/Delta IV Heavy & 56 & 212 \\
\hline KSC/Falcon 9 v1.1 & 56 & 303 \\
\hline Baikonur/Proton-M & 141 & 597 \\
\hline
\end{tabular}

from the same spaceport due to bigger inclination change during first burst in Falcon 9 mission characteristics. For the following calculations with Kennedy space center only more efficient Hohmann transfer will be used except for Falcon 9 launch.

\section{Results}

Even though it is not absolutely precise, because there are still forces acting against rocket which are not included in this work, spaceports impact on rocket mission efficiency can be determined. After all calculations needed to get decent picture of space missions to LEO and GEO we can interpret results. Obviously Zenit rocket and Sea Launch platform are not shown in the results, because all shifting to different spaceport is theoretically made to Equator, thus Sea Launch, because it is the most efficient. Zenit rocket launches are so far not economically efficient to shift somewhere else, because its home spaceport is the best. So changing spaceports for missions to LEO has this following effect described in Tab. 6.

As it is clearly visible that Delta IV Heavy has the same speed difference, increase in payload is possible bigger for Falcon 9, because it is new rocket with more efficient performance. 
It is absolutely clear that changing spaceports and building new support facilities is not good option even for the biggest gain for Proton-M missions.

On the other hand as there are results for GEO missions with Hohmann transfer in Tab. 7. huge differences are plainly visible. Good thing is that difference between Sea Launch and Guyana Space Center is so small that we can think about this numbers the same for changing spaceport to GSC. It is obvious that it is much better to build support facilities and specific launch pad for each rocket on the ground than to rebuild oil mining platform to accommodate different rockets or rebuild new ones for each rocket.

Table 6. Differences for spaceport change.

\begin{tabular}{lrr} 
Spaceport/Rockets & $\begin{array}{c}\Delta \mathbf{v} \text { difference } \\
\left(\mathbf{m s}^{-1}\right)\end{array}$ & $\begin{array}{c}\text { Payload } \\
\text { increase }(\mathbf{k g})\end{array}$ \\
\hline GSC/Ariane 5 & 0.48 & 2 \\
\hline KSC/Delta IV Heavy & 398.81 & 869 \\
\hline KSC/Falcon 9 v1.1 & 420.55 & 1544 \\
\hline Baikonur/Proton-M & 1061.35 & 2571 \\
\hline
\end{tabular}

Table 7. Hohmann transfers from home spaceport.

\begin{tabular}{lr}
\multicolumn{1}{c}{ Rocket } & $\Delta v\left(\mathbf{k m ~ s}^{-1}\right)$ \\
\hline Ariane 5 & 3.930 \\
\hline Delta IV Heavy & 4.274 \\
\hline Falcon 9 v1.1 & 4.296 \\
\hline Proton-M & 4.852 \\
\hline Zenit & 3.931 \\
\hline
\end{tabular}

Both American rockets starting from Kennedy Space Center get very nice payload bonus when moved closer to the Equator. Falcon 91.5 tones increase is huge, and worthy to think about changing home spaceport especially because no government agency is owner of this rocket.

These results also shows that Baikonur spaceport is very inefficient for GEO missions and it supports the latest development, where Russian space agency (Roskosmos) and its commercial branch responsible for Soyuz rocket launches for commercial purposes is building support facilities and launch pad in Guyana Space Complex. This change of home spaceport for Soyuz rocket even though we have results only for Proton-M will surely have huge impact on economical efficiency on Soyuz missions.

\section{Conclusion}

Calculations made in this paper clearly show that missions to LEO and other low orbits are not needed to be repositioned to other spaceports. But future missions will go much further than just to low orbits. Missions to asteroids, other planet and to the deep space are in development and since no new engines are in sight in near future and we are stuck for now with current technology, it is necessary to rethink our spaceport policy.
Unfortunately space missions are very connected to national policy, international image and last but not least secret army projects. Therefore multinational spaceports used for international cooperation in space exploration will be very hard to build and even harder to be working properly.

\section{Acknowledgments}

This paper was prepared as output of project work on project Space technology in master study program.

\section{References}

[1] R.R. Bate, D.D. Mueller, and J.E. White. Fundamentals of Astrodynamics (Dover Books on Aeronautical Engineering). Dover Publications, 1971. ISBN 0486600610.

[2] Arianespace, service\&solutions. Ariane 5: User's Manual, 2011. URL http://www.arianespace.com/ wp-content/uploads/2015/09/Ariane5_ users_manual_Issue5_July2011.pdf. [Online].

[3] Boeing. Delta IV Payload Planners Guide, 1999. URL http: / / spacecraft.ssl .umd.edu/design_ lib/Delta4.pl.guide.pdf. [Online].

[4] SpaceX. Falcon 9 Launch Vehicle: Payload User's guide, 2015. URL http://www.spacex.com/sites/ spacex/files/falcon_9_users_guide_rev_ 2.0.pdf. [Online].

[5] International Launch Services. Proton Launch System: Mission Planner's Guide, 2009. URL http: //www . ilslaunch. com/node/42. [Online].

[6] Center for Operation of Ground-based Space Infrastructure Facilities. Zenit-3SLBF ILV/Zenit-M SRC User's guide, 2011. URL http://www.tsenki.com/en/ launch_services/equipment_maintenance/ zenit_3slbf/guide/users_guide.pdf. [Online].

[7] W. Hohmann. The Attainability of Heavenly Bodies. NASA, 1960. 